\title{
GENERALISED NETWORK DESCRIPTORS
}

\author{
Suzana Antunović, Tonći Kokan, Tanja Vojković and Damir \\ VUKIČEVIĆ \\ University of Split, Croatia
}

\begin{abstract}
Transmission and betweenness centrality are key concepts in communication networks theory. Based on this concept, new concepts of networkness and network surplus have recently been defined. However, all these four concepts include unrealistic assumption about equal communication between vertices. Here, we propose more realistic assumption that the amount of communication of vertices decreases as their distance increases. We assume that amount of communication between vertices $u$ and $v$ is proportional to $d(u, v)^{\lambda}$ where $\lambda<0$. Taking this into account generalised versions of these four descriptors are defined. Extremal values of these descriptors are analysed.
\end{abstract}

\section{INTRODUCTION}

Complex networks $([2,7,15])$ are extensively used to model objects and their relations in many situations. They are very often used in communication theory ([3]). We restrict our attention on the representation of a complex network as a simple connected graph $G=(V, E)$. Throughout this paper, we use standard graph-theoretical terminology $([4])$.

Standard vertex betweenness (as defined firstly in [9] and [10]) is efficiently computed by algorithm of Brandes [6] and studied in [11]. It has been applied in complex networks to indicate importance of the corresponding node (person) $([1,9])$. The edge betweenness, a similar concept, was first introduced by Girvan and Newman in [12] and used in the connection with community detection in complex networks.

2010 Mathematics Subject Classification. 05C35, 05C12.

Key words and phrases. Betweenness, transmission, networkness, network surplus, complex networks. 
For an edge $u v$, edge betweenness $b(u v)$ is defined in the following way

$$
b(u v)=\sum_{\{k, l\} \in\left(\begin{array}{c}
V \\
2
\end{array}\right)} \frac{s_{u v}^{k l}}{s^{k l}},
$$

where $s_{u v}^{k l}$ is the number of shortest paths between vertices $k$ and $l$ that pass through the edge $u v$ and $s^{k l}$ is the total number of shortest paths between $k$ and $l$.

Betweenness centrality $c(u)$ of a vertex $u$ is sum of edge betweennesses of all edges incident to $u$ :

$$
c(u)=\sum_{v \in[u]} b(u v),
$$

where $[u]$ is the set of neighbours of vertex $u([8])$.

Note that measure is closely related to, yet different from Freeman's betweenness centrality $b$. It holds that $([16])$

$$
b(u)=c(u)-n+1 .
$$

because Freeman's betweenness centrality does not take into account $n-1$ paths that start in vertex $u$.

Moreover, in the context of the communication networks betweenness centrality $c(u)$ can be reinterpreted as the quantity of communication processed by a node $u([16])$. On the other hand, transmission (also called farness, status of total distance) of the vertex $u$

$$
t(u)=\sum_{v \in V} d(u, v),
$$

where $d(u, v)$ is the distance between vertices $u$ and $v$, can be interpreted as the cost of the vertex to the network $([16])$. Also note that transmission is the inverse of closeness centrality $([10])$ and that transmission is characteristic path length multiplied by $n-1$.

It is well-known that both these quantities are connected with Wiener index ([17]) defined by

$$
W(G)=\frac{1}{2} \sum_{(u, v) \in V^{2}} d(u, v) .
$$

Namely, as proven in [8] it holds that

$$
\sum_{u \in V} c(u)=\sum_{u \in V} t(u)=2 W(G) .
$$

Network surplus of the vertex $u$ ("added value" to the network provided by vertex $u$ ) is defined by $\nu(u)=c(u)-t(u)$. Another way to measure "productivity" of vertex $u$ is its networkness ([16]) defined by $N(u)=$ $c(u) / t(u)$. 
Note that interpretation of the betweenness centrality as the amount of information processed by the vertex $u$ assumes that the quantity of the information exchanged by any two vertices is the same. Here, we amend this by weighting the amount of communication by $d(u, v)^{\lambda}$ for some $\lambda<0$, generalising the case $\lambda=-1$ introduced in [5] under the name scaled betweenness. For an arbitrary $\lambda \in \mathbb{R}$, we define

$$
\begin{aligned}
t_{\lambda}(u) & =\sum_{v \in V \backslash\{u\}} d(u, v) \cdot d(u, v)^{\lambda}=\sum_{v \in V \backslash\{u\}} d(u, v)^{1+\lambda}, \\
b_{\lambda}(u v) & =\sum_{\{k, l\} \in\left(\begin{array}{c}
V \\
2
\end{array}\right)} \frac{s_{u v}^{k l}}{s^{k l}} \cdot d(k, l)^{\lambda}, \\
c_{\lambda}(u) & =\sum_{v \in[u]} b_{\lambda}(u v) .
\end{aligned}
$$

Note that the function $t_{\lambda}$ has a threshold in $\lambda=-1$ since it is increasing function in terms of distances for $\lambda>-1$ and decreasing for $\lambda<-1$.

We define

$$
\begin{aligned}
N_{\lambda}(u) & =\frac{c_{\lambda}(u)}{t_{\lambda}(u)}, \\
\nu_{\lambda}(u) & =c_{\lambda}(u)-t_{\lambda}(u) .
\end{aligned}
$$

Note that, by putting $\lambda=0$, we get standard definitions of betweenness centrality, transmission, networkness and network surplus $([8,16])$. In this paper we restrict ourselves to the case $\lambda<0$ because positive lambdas lead to unrealistic assumption that distant vertices communicate more than the close ones.

Analogously as in [16] we define

$$
\begin{aligned}
m c_{\lambda}(G) & =\min \left\{c_{\lambda}(u): u \in V\right\}, \\
M c_{\lambda}(G) & =\max \left\{c_{\lambda}(u): u \in V\right\}, \\
m t_{\lambda}(G) & =\min \left\{t_{\lambda}(u): u \in V\right\}, \\
M t_{\lambda}(G) & =\max \left\{t_{\lambda}(u): u \in V\right\}, \\
m N_{\lambda}(G) & =\min \left\{N_{\lambda}(u): u \in V\right\}, \\
M N_{\lambda}(G) & =\max \left\{N_{\lambda}(u): u \in V\right\}, \\
m \nu_{\lambda}(G) & =\min \left\{\nu_{\lambda}(u): u \in V\right\}, \\
M \nu_{\lambda}(G) & =\max \left\{\nu_{\lambda}(u): u \in V\right\} .
\end{aligned}
$$

and we are interested in finding lower and upper bounds of these values for all $\lambda<0$.

Our results can be summarised in Tables 1 and 2 . 


\begin{tabular}{|c|c|c|}
\hline Descriptor & \multicolumn{2}{|c|}{$\lambda \in\langle-\infty,-1\rangle$} \\
\hline & Lower bound & Upper bound \\
\hline \multirow[t]{2}{*}{$m t_{\lambda}$} & path (end vertices) & complete graph \\
\hline & $\sum_{i=1}^{n-1} i^{1+\lambda}$ & $n-1$ \\
\hline \multirow[t]{2}{*}{$M t_{\lambda}$} & path (center) & complete graph \\
\hline & $\begin{array}{ll}2 \cdot \sum_{i=1}^{\frac{n-1}{2}} i^{1+\lambda}, & n \text { odd } \\
\sum_{i=1}^{\frac{n}{2}} i^{1+\lambda}+\sum_{i=1}^{\frac{n}{2}-1} i^{1+\lambda}, & n \text { even }\end{array}$ & $n-1$ \\
\hline \multirow[t]{2}{*}{$m c_{\lambda}$} & path (end vertices) & complete graph \\
\hline & $\sum_{i=1}^{n-1} i^{\lambda}$ & $n-1$ \\
\hline \multirow[t]{2}{*}{$M c_{\lambda}$} & open problem & star (center) \\
\hline & & $n-1+(n-1)(n-2) 2^{\lambda}$ \\
\hline \multirow[t]{2}{*}{$m N_{\lambda}$} & broom (starting vertex) & vertex-transitive graph \\
\hline & 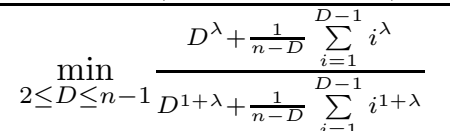 & 1 \\
\hline \multirow[t]{2}{*}{$M N_{\lambda}$} & vertex-transitive graph & star (center) \\
\hline & 1 & $2^{\lambda}(n-2)+1$ \\
\hline \multirow[t]{2}{*}{$m \nu_{\lambda}$} & star (pendant vertex) & vertex-transitive graph \\
\hline & $-(n-2) 2^{\lambda}$ & 0 \\
\hline \multirow[t]{2}{*}{$M \nu_{\lambda}$} & vertex-transitive graph & star (center) \\
\hline & 0 & $(n-1)(n-2) 2^{\lambda}$ \\
\hline
\end{tabular}

TABLE 1

\section{Generalised Wiener index}

Similarly to the case $\lambda=0$, the following holds:

LEMma 2.1. For each graph $G=(V, E)$

$$
\sum_{u \in V} t_{\lambda}(u)=\sum_{u \in V} c_{\lambda}(u)=2 W_{1+\lambda}(G) .
$$

Proof. For transmission, this statement follows directly from the definition. Let us prove the statement for betweenness centrality. We have

$$
\sum_{u \in V} c_{\lambda}(u)=\sum_{u \in V} \sum_{v \in[u]} \sum_{\{k, l\} \in\left(\begin{array}{c}
V \\
2
\end{array}\right)} \frac{s_{u v}^{k l}}{s^{k l}} d(k, l)^{\lambda}=\sum_{\{k, l\} \in\left(\begin{array}{c}
V \\
2
\end{array}\right)} \frac{d(k, l)^{\lambda}}{s^{k l}} \sum_{u \in V} \sum_{v \in[u]} s_{u v}^{k l} .
$$




\begin{tabular}{|c|c|c|}
\hline Descriptor & \multicolumn{2}{|c|}{$\lambda \in\langle-1,0\rangle$} \\
\hline & Lower bound & Upper bound \\
\hline \multirow[t]{2}{*}{$m t_{\lambda}$} & complete graph & path (center) \\
\hline & $n-1$ & $\begin{array}{ll}2 \cdot \sum_{i=1}^{\frac{n-1}{2}} i^{1+\lambda}, & n \text { odd } \\
\sum_{i=1}^{\frac{n}{2}} i^{1+\lambda}+\sum_{i=1}^{\frac{n}{2}-1} i^{1+\lambda}, & n \text { even }\end{array}$ \\
\hline \multirow[t]{2}{*}{$M t_{\lambda}$} & complete graph & path (end vertices) \\
\hline & $n-1$ & $\sum_{i=1}^{n-1} i^{1+\lambda}$ \\
\hline \multirow[t]{2}{*}{$m c_{\lambda}$} & path (end vertices) & open problem \\
\hline & $\sum_{i=1}^{n-1} i^{\lambda}$ & \\
\hline \multirow[t]{2}{*}{$M c_{\lambda}$} & complete graph & star (center) \\
\hline & $n-1$ & $n-1+(n-1)(n-2) 2^{\lambda}$ \\
\hline \multirow[t]{2}{*}{$m N_{\lambda}$} & path (end vertices) & vertex-transitive graph \\
\hline & $\frac{\sum_{i=1}^{n-1} i^{\lambda}}{\sum_{i=1}^{n-1} i^{1+\lambda}}$ & 1 \\
\hline \multirow[t]{2}{*}{$\overline{M N_{\lambda}}$} & vertex-transitive graph & star (center) \\
\hline & 1 & $2^{\lambda}(n-2)+1$ \\
\hline \multirow[t]{2}{*}{$m \nu_{\lambda}$} & path (end vertices) & vertex-transitive graph \\
\hline & $\sum_{i=1}^{n-1}\left(i^{\lambda}-i^{1+\lambda}\right)$ & 0 \\
\hline \multirow[t]{2}{*}{$M \nu_{\lambda}$} & vertex-transitive graph & star (center) \\
\hline & 0 & $(n-1)(n-2) 2^{\lambda}$ \\
\hline
\end{tabular}

TABLE 2

For a given pair of vertices $\{k, l\}$,

$$
\sum_{u \in V} \sum_{v \in[u]} s_{u v}^{k l}
$$

is the number of pairs $(u, v)$ of vertices such that $d(u, v)=1$ and that a shortest path between $k$ and $l$ passes through the edge $u v$. The length of each of the $s^{k l}$ shortest paths from $k$ to $l$ is $d(k, l)$ and therefore on each such path we can choose $d(k, l)$ pairs $\{u, v\}$ such that $d(u, v)=1$. Thus,

$$
\sum_{u \in V} \sum_{v \in[u]} s_{u v}^{k l}=2 d(k, l) \cdot s^{k l} .
$$


Finally,

$$
\sum_{u \in V} c_{\lambda}(u)=\sum_{\{k, l\} \in\left(\begin{array}{c}
V \\
2
\end{array}\right)} \frac{d(k, l)^{\lambda}}{s^{k l}} \cdot 2 d(k, l) \cdot s^{k l}=2 \sum_{\{k, l\} \in\left(\begin{array}{c}
V \\
2
\end{array}\right)} d(k, l)^{1+\lambda}=2 W_{1+\lambda}(G) .
$$

\section{TRANSMISSION}

We first discuss $t_{\lambda}(u)=\sum_{v \in V \backslash\{u\}} d(u, v)^{1+\lambda}$ for $\lambda<0$. Obviously,

$$
M t_{-1}(G)=m t_{-1}(G)=n-1,
$$

for each graph $G$ with $n$ vertices.

\subsection{Minimal transmission.}

THEOREM 3.1. For $\lambda \in\langle-\infty,-1\rangle$ and for each graph $G$ with $n$ vertices it holds that

$$
\sum_{i=1}^{n-1} i^{1+\lambda} \leqslant m t_{\lambda}(G) \leqslant n-1
$$

The lower bound is reached for a path (in its end-vertices), while the upper bound is reached for a complete graph.

Proof. The upper bound follows simply from the fact that each summand in $\sum_{v \in V \backslash\{u\}} d(u, v)^{1+\lambda}$ is at most 1 and equality holds in a complete graph.

Let us prove the lower bound. The sum increases when any summand increases, i.e., when any distance $d(u, v)$ decreases, so the lower bound of $m t_{\lambda}$ will be reached for a tree (by adding edges on a tree we can only decrease distances). Let $G$ be a tree for which $m t_{\lambda}(G)$ is minimal and let $u_{0}$ be a vertex such that $t_{\lambda}\left(u_{0}\right)=m t_{\lambda}(G)$. We will prove that $G$ is a path and that $u_{0}$ is an end-vertex of that path. Assume that this it not the case. That means that either $G$ is not a path or $u_{0}$ is not an end-vertex of the path. In both cases, there are at least two leaves in $V \backslash\left\{u_{0}\right\}$, we denote them by $u_{1}$ and $u_{2}$.

Without loss of generality, we can assume $d\left(u_{0}, u_{1}\right) \leqslant d\left(u_{0}, u_{2}\right)$. Now let us observe the graph $G^{\prime}$ that is obtained from $G$ by cutting the leaf $u_{1}$ from its place and putting it on the leaf $u_{2}$. It is obvious that the value of $t_{\lambda}\left(u_{0}\right)$ is smaller in $G^{\prime}$ than in $G$, because $u_{1}$ is farther from $u_{0}$ in $G^{\prime}$ and that contradicts the assumption that $G$ has the minimal value of $t_{\lambda}(G)$. Therefore, $u_{0}$ is an end-vertex of the path $G=P_{n}$. Now the bound is easily calculated

$$
m t_{\lambda}(G) \geqslant m t_{\lambda}\left(P_{n}\right)=\sum_{i=1}^{n-1} i^{1+\lambda}
$$


TheOREM 3.2. For each graph $G$ and $\lambda \in\langle-1,0\rangle$, it holds

$$
n-1 \leqslant m t_{\lambda}(G) \leqslant\left\{\begin{array}{cc}
2 \cdot \sum_{i=1}^{\frac{n-1}{2}} i^{1+\lambda}, & n \text { odd } \\
\sum_{i=1}^{\frac{n}{2}} i^{1+\lambda}+\sum_{i=1}^{\frac{n}{2}-1} i^{1+\lambda}, & n \text { even }
\end{array}\right\}
$$

where the upper bound is reached for a path (in its central vertex) and the lower bound for a complete graph.

Proof. The lower bound is trivial. Let us prove the upper bound. We conclude that the upper bound is reached for a tree, since we have to maximize the distances (note that adding edges decreases distances and hence decreases $\left.m t_{\lambda}\right)$. We will prove that such tree is a path and the vertex in a path with minimal value of $t_{\lambda}$ is its center (or two centers, if $n$ is even). In a path $P_{n}$ it obviously holds

$$
m t_{\lambda}\left(P_{n}\right)=\left\{\begin{array}{ll}
2 \cdot \sum_{i=1}^{\frac{n-1}{2}} i^{1+\lambda}, & n \text { odd } \\
\sum_{i=1}^{\frac{n}{2}} i^{1+\lambda}+\sum_{i=1}^{\frac{n}{2}-1} i^{1+\lambda}, & n \text { even }
\end{array}\right\} .
$$

Let $G$ be any tree with $n$ vertices, and let $c \in V$ be a center, i.e. let $c$ be a vertex of graph $G$ such that $\max \{d(c, u): u \in V\}$ is minimal. It is a well-known fact that every tree either has one center or two adjacent centers.

Obviously it follows that $d(c, u) \leqslant\left\lfloor\frac{n}{2}\right\rfloor$, for every $u \in V$.

From the definition of $m t_{\lambda}(G)$ we know that $m t_{\lambda}(G) \leqslant t_{\lambda}(c)$ and our aim is to prove that $t_{\lambda}(c) \leqslant m t_{\lambda}\left(P_{n}\right)$.

For $v \in V$, let $S(v)$ denote the unique path from $c$ to $v$, for $v \in V$. Let $u_{1}$ be a vertex that is farthest from $c$. Furthermore, let $u_{2}$ be a farthest vertex from $c$ that satisfies the condition that $S\left(u_{1}\right) \cap S\left(u_{2}\right)=\emptyset$. Obviously, both $u_{1}$ and $u_{2}$ are leafs.

Since $c$ is a center of the graph, one of the following holds

$$
d\left(u_{1}, c\right)=d\left(u_{2}, c\right) \quad \text { or } \quad d\left(u_{1}, c\right)=d\left(u_{2}, c\right)+1 .
$$

Now, we will move all the other vertices in $G$, not contained in neither $S\left(u_{1}\right)$ nor $S\left(u_{2}\right)$ to the ends of those paths.

In each step we move one leaf from $G \backslash\left(S\left(u_{1}\right) \cup S\left(u_{2}\right)\right)$ to the end of either $S\left(u_{1}\right)$ or $S\left(u_{2}\right)$ (Figure 1). We put the first leaf on $S\left(u_{2}\right)$ and then proceed by alternating paths in each step until we are left with graph $G^{\prime}=S_{1} \cup S_{2}$.

The final graph will obviously be a path and since the condition (3.1) is satisfied throughout the process, vertex $c$ will also be a center of $G^{\prime}$.

Every time we move a vertex, its distance from the center $c$ either becomes larger or stays the same, and thus the value of $t_{\lambda}(c)$ either increases or stays 

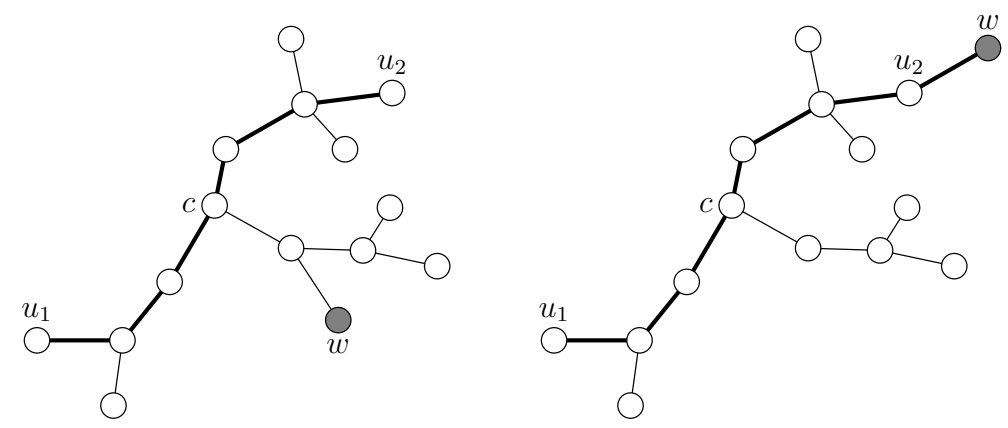

FIGURE 1. Relocating leaf $w$.

the same. Thus we have

$$
m t_{\lambda}(G) \leqslant t_{\lambda}(c) \leqslant m t_{\lambda}\left(G^{\prime}\right)=m t_{\lambda}\left(P_{n}\right),
$$

so the claim is proven.

\subsection{Maximal transmission}

THEOREM 3.3. For each graph $G$ with $n$ vertices and for $\lambda \in\langle-\infty,-1\rangle$ the following holds

$$
\left\{\begin{array}{cc}
2 \cdot \sum_{i=1}^{\frac{n-1}{2}} i^{1+\lambda}, & n \text { odd } \\
\sum_{i=1}^{\frac{n}{2}} i^{1+\lambda}+\sum_{i=1}^{\frac{n}{2}-1} i^{1+\lambda}, & n \text { even }
\end{array}\right\} \leqslant M t_{\lambda}(G) \leqslant n-1
$$

where the upper bound is reached for a complete graph and the lower bound for a path (in its center).

Proof. This proof is analogous to the proof of the Theorem 3.2.

THEOREM 3.4. For each graph $G$ with $n$ vertices and for $\lambda \in\langle-1,0\rangle$, the following holds

$$
n-1 \leqslant M t_{\lambda}(G) \leqslant \sum_{i=1}^{n-1} i^{1+\lambda} .
$$

The lower bound is obtained for a complete graph and the upper bound is obtained for a path (in its end-vertex).

Proof. The proof is analogous to the proof of the Theorem 3.1. 


\section{Betweenness Centrality}

We will need the following auxiliary lemma.

LEMMA 4.1. For all $\lambda<0$ and for a given integer $n$, among all graphs with $n$ vertices, any graph $G$ for which maximum $c_{\lambda}(G)$ is obtained is a tree.

Proof. Let $G$ be a graph such that $c_{\lambda}(G)$ is maximal and let $u$ be a vertex for which maximal betweenness centrality is reached. We will prove that $G$ is a tree.

Suppose that is not the case. Let us introduce a spanning tree $G^{\prime}$ of $G$ obtained as follows: starting from vertex $u$, in each step we choose a vertex $v$ that is closest to $u$ (the distance between $u$ and $v$ is minimal) and is still outside the tree. Since $G^{\prime}$ is a tree, it holds that $\frac{s_{u v}^{k l}}{s^{k l}}=1$ for each $k, l \in V$ that are connected by a path passing through the edge $u v$. From the way $G^{\prime}$ was obtained, it is obvious that the distances between $u$ and $v$, for every $v \in V$ will stay the same. This means that $c_{\lambda}(u)$ is greater in $G^{\prime}$ than in $G$ which contradicts our assumption.

\subsection{Minimal betweenness centrality.}

THEOREM 4.2. For each graph $G$ with $n$ vertices and for $\lambda<0$ it holds that

$$
m c_{\lambda}(G) \geqslant \sum_{i=1}^{n-1} i^{\lambda} .
$$

The equality holds for a path (in its end-vertex).

Proof. Let $G$ be a graph for which $m c_{\lambda}(G)$ is minimal and let $u$ be a vertex such that $c_{\lambda}(u)=m c_{\lambda}(G)$. Note that

$$
m c_{\lambda}(G) \geqslant \sum_{v \in V \backslash\{u\}} d(u, v)^{\lambda}
$$

because the sum on the right-hand side corresponds to contributions of paths starting in $u$.

From Theorem 3.1, because $\lambda-1<-1$, we have

$$
\begin{aligned}
m c_{\lambda}(G) & \geqslant \sum_{v \in V \backslash\{u\}} d(u, v)^{\lambda}=t_{\lambda-1}(u) \\
& \geqslant \sum_{i=1}^{n-1} i^{(\lambda-1)+1}=\sum_{i=1}^{n-1} i^{\lambda} .
\end{aligned}
$$

THEOREM 4.3. For each graph $G$ with $n$ vertices and for $\lambda \in\langle-\infty,-1\rangle$ it holds that

$$
m c_{\lambda}(G) \leqslant n-1 .
$$


The equality holds for a complete graph.

Proof. For a given graph $G$ the average betweenness centrality of all vertices can be bounded, using Lemma 2.1 and the fact that $1+\lambda<0$, in the following way

$$
\frac{1}{n} \sum_{u \in V} c_{\lambda}(u)=\frac{2}{n} W_{1+\lambda}(G)=\frac{2}{n} \sum_{\{k, l\} \in\left(\begin{array}{c}
V \\
2
\end{array}\right)} d(k, l)^{1+\lambda} \leqslant \frac{2}{n} \sum_{\{k, l\} \in\left(\begin{array}{c}
V \\
2
\end{array}\right)} 1=n-1 .
$$

Since the minimal betweenness centrality is less than or equal to the average betweenness centrality, our claim is proven. The equality holds if and only if $d(k, l)=1$ for all pairs $k, l$, that is, exactly in the case when $G$ is a complete graph.

Finding $m c_{\lambda}(G)$ for $\lambda \in\langle-1,0\rangle$ seems to be much harder problem. We solve it only in the special case of 2 -connected graph. In general case we conjecture.

CONJECTURE 4.4. For each graph $G$ with $n \geqslant 3$ vertices and for $\lambda \in$ $\langle-1,0\rangle$ it holds that

$$
m c_{\lambda}(G) \leqslant\left\{\begin{array}{ll}
2 \cdot \sum_{i=1}^{\frac{n-1}{2}} i^{1+\lambda}, & n \text { odd } \\
2 \cdot \sum_{i=1}^{\frac{n-2}{2}} i^{1+\lambda}+\left(\frac{n}{2}\right)^{1+\lambda}, & n \text { even }
\end{array}\right\}
$$

The equality holds for a cycle.

REMARK 4.5. The previous conjecture is true in the special case when $G$ is a $2-$ connected graph. To prove this we need the following lemma.

Lemma 4.6. Let $n \geqslant 3$, let $\lambda \in\langle-1,0\rangle$ and let $S$ be a set of sequences $\left(x_{1}, x_{2}, \ldots, x_{\lfloor n / 2\rfloor}\right) \in \mathbb{N}^{\lfloor n / 2\rfloor}$ such that $x_{1}+x_{2}+\ldots+x_{\lfloor n / 2\rfloor}=n-1$ and there exists $k \in\{1, \ldots,\lfloor n / 2\rfloor\}$ such that $x_{i} \geq 2$ for each $i \leq k$ and $x_{i}=0$ for each $i>k$. Let $S^{\prime}$ be the set of sequences in $S$ of the form $\left(x_{1}, x_{2}, \ldots, x_{\lfloor n / 2\rfloor}\right)$ such that there is $k \in\{1, \ldots,\lfloor n / 2\rfloor\}$ such that $x_{k} \in\{0,1\}, x_{i}=2$ for each $1 \leq i<k$ and $x_{i}=0$ for each $i>k$. Let $T_{n}$ be defined by

$$
T_{n}\left(x_{1}, x_{2}, \ldots, x_{\lfloor n / 2\rfloor}\right)=\sum_{i=1}^{\lfloor n / 2\rfloor} x_{i} \cdot i^{1+\lambda} .
$$

Then

$$
\max \left\{T_{n}(s): s \in S\right\}=\max \left\{T_{n}(s): s \in S^{\prime}\right\} .
$$


Furthermore, maximal value $M_{n}$ of $T_{n}$ in $S^{\prime}$ is:

$$
\begin{aligned}
& 2 \cdot \sum_{i=1}^{\frac{n-1}{2}} i^{1+\lambda}, \quad n \text { odd; } \\
& 2 \cdot \sum_{i=1}^{\frac{n-2}{2}} i^{1+\lambda}+\left(\frac{n}{2}\right)^{1+\lambda}, \quad n \text { even. }
\end{aligned}
$$

Proof. Suppose to the contrary. Let $\left(x_{1}, x_{2}, \ldots, x_{\lfloor n / 2\rfloor}\right) \notin S^{\prime}$ maximize $T_{n}$ in $S$. Then there is $k$ such that $x_{k}>2$. Note that $k<\lfloor n / 2\rfloor$. Then,

$$
\left(x_{1}, x_{2}, \ldots, x_{k}-1, x_{k+1}+1, x_{k+2}, \ldots, x_{\lfloor n / 2\rfloor}\right) \in S .
$$

It follows that

$$
\begin{aligned}
0 & \leq T_{n}\left(x_{1}, x_{2}, \ldots, x_{\lfloor n / 2\rfloor}\right)-T_{n}\left(x_{1}, x_{2}, \ldots, x_{k}-1, x_{k+1}+1, x_{k+2}, \ldots, x_{\lfloor n / 2\rfloor}\right) \\
& =k^{1+\lambda}-(k+1)^{1+\lambda}<0,
\end{aligned}
$$

which is a contradiction. So the sequence $s \in S^{\prime}$ that maximizes $T_{n}$ is $(2,2, \ldots, 2,0)$ for $n$ odd, and $(2,2, \ldots 2,1)$ for $n$ even. It can be easily seen that the value of $T_{n}$ for those sequences is $2 \cdot \sum_{i=1}^{\frac{n-1}{2}} i^{1+\lambda}$ in the first case, and $2 \cdot \sum_{i=1}^{\frac{n-2}{2}} i^{1+\lambda}+\left(\frac{n}{2}\right)^{1+\lambda}$ in the second case.

Proof of Remark 4.5. Let us denote the right-hand side of the inequality (4.1) by $\operatorname{cyc}_{\lambda}(n)$ and assume the contrary - that there exists a 2 -connected graph $G$ with $n$ vertices such that $m c_{\lambda}(G)>\operatorname{cyc}_{\lambda}(n)$. This implies that $c_{\lambda}(u)>\operatorname{cyc}_{\lambda}(n)$, for all $u \in V$. Therefore

$$
\sum_{u \in V} t_{\lambda}(u)=2 W_{1+\lambda}(G)=\sum_{u \in V} c_{\lambda}(u)>n \cdot \operatorname{cyc}_{\lambda}(n),
$$

and thus there exists $w \in V$ such that $t_{\lambda}(w)>\operatorname{cyc}_{\lambda}(n)$.

Let $w_{1}$ be the vertex that is farthest from $w$ and let $d\left(w, w_{1}\right)=D$. Since $G$ is 2 -connected it holds that for every $d<D$ there are at least 2 vertices on a distance $d$ from $w$. From that it is easily seen that $D \leq\left\lfloor\frac{n}{2}\right\rfloor$. Let us denote by $x_{i}$ the number of vertices on a distance $i$ from $w$, and let us observe the sequence $\left(x_{1}, \ldots, x_{\lfloor n / 2\rfloor}\right)$. This sequence is obviously in $S$ defined in Lemma 4.6 .

It follows $t_{\lambda}(w) \leq \operatorname{cyc}(n)$, which is a contradiction.

REMARK 4.7. In another special case, when there exists a vertex $u_{0}$ such that no shortest path between some vertices different from $u_{0}$ passes through $u_{0}$, strict inequality holds in Conjecture 4.4. 
Proof. Note that

$$
c_{\lambda}\left(u_{0}\right)=\sum_{v \in V \backslash\left\{u_{0}\right\}} d\left(u_{0}, v\right)^{\lambda} \leqslant n-1
$$

because only paths starting in $u_{0}$ contibute to $c_{\lambda}\left(u_{0}\right)$. Hence

$$
m c_{\lambda}(G) \leqslant c_{\lambda}\left(u_{0}\right)=\sum_{v \in V \backslash\left\{u_{0}\right\}} d\left(u_{0}, v\right)^{\lambda} \leqslant n-1<\operatorname{cyc}_{\lambda}(n) .
$$

\subsection{Maximal betweenness centrality.}

THEOREM 4.8. For each graph $G$ with $n$ vertices and for $\lambda<0$ it holds that

$$
M c_{\lambda}(G) \leqslant n-1+(n-1)(n-2) \cdot 2^{\lambda} .
$$

The bound is reached for a star (in its central vertex).

Proof. Let $G$ be a graph such that $c_{\lambda}(G)$ is maximal and let $u_{0}$ be a vertex such that $c_{\lambda}\left(u_{0}\right)=M c_{\lambda}(G)$. If there exists a vertex $v \in V \backslash\left\{u_{0}\right\}$ such that $u_{0}$ and $v$ are not adjacent, then adding edge $u_{0} v$ would increase $c_{\lambda}\left(u_{0}\right)$ because some distances would become smaller (thus increasing betweenness centrality) and fractions $s_{u_{0} v}^{k l} / s^{k l}$ would either stay the same or increase. This contradicts our assumption of maximality of $c_{\lambda}\left(u_{0}\right)$ which means that $u_{0}$ is connected to every other vertex in the graph.

If there are vertices $v, w \in V \backslash\left\{u_{0}\right\}$ that are adjacent then deleting the edge $v w$ would increase $c_{\lambda}\left(u_{0}\right)$ in a similar way as before. Therefore, $G$ is a star with $u_{0}$ as its center. Finally, we have

$$
\begin{aligned}
M c_{\lambda}\left(S_{n}\right) & =c_{\lambda}\left(u_{0}\right)=\sum_{v \in N\left(u_{0}\right) k, l \in V} \sum_{u_{u_{0} v}^{k l}} \cdot d(k, l)^{\lambda} \\
& =\sum_{v \in V \backslash\left\{u_{0}\right\}} d\left(u_{0}, v\right)^{\lambda}+2 \sum_{v, w \in V \backslash\left\{u_{0}\right\}} d(v, w)^{\lambda} \\
& =n-1+(n-1)(n-2) \cdot 2^{\lambda} .
\end{aligned}
$$

Conjecture 4.9. For each graph $G$ with $n$ vertices and for $\lambda \in\langle-\infty,-1\rangle$ it holds that

$$
M c_{\lambda}(G) \geqslant\left\{\begin{array}{ll}
2 \cdot \sum_{i=1}^{\frac{n-1}{2}} i^{1+\lambda}, & n \text { odd } \\
2 \cdot \sum_{i=1}^{\frac{n-2}{2}} i^{1+\lambda}+\left(\frac{n}{2}\right)^{1+\lambda}, & n \text { even }
\end{array}\right\} .
$$

The equality holds for a cycle. 
REMARK 4.10. Special cases analogous to Remarks 4.5 and 4.7 can be proven in a similar way.

THEOREM 4.11. For each graph $G$ with $n$ vertices and for $\lambda \in\langle-1,0\rangle$ it holds that

$$
M c_{\lambda}(G) \geqslant n-1 \text {. }
$$

The equality holds for a complete graph.

Proof. For a given graph $G$ the average betweenness centrality of all vertices can be bounded, using Lemma 2.1 and the fact that $1+\lambda>0$, in the following way

$$
\frac{1}{n} \sum_{u \in V} c_{\lambda}(u)=\frac{2}{n} W_{1+\lambda}(G)=\frac{2}{n} \sum_{\{k, l\} \in\left(\begin{array}{c}
V \\
2
\end{array}\right)} d(k, l)^{1+\lambda} \geqslant \frac{2}{n} \sum_{\{k, l\} \in\left(\begin{array}{c}
V \\
2
\end{array}\right)} 1=n-1 .
$$

Since the maximal betweenness centrality is greater than or equal to the average betweenness centrality, our claim is proven. In a complete graph $d(u, v)=1$ for all vertices $u \neq v$ and thus the equality holds for a complete graph.

\section{NeTWORKNESS}

5.1. Minimal networkness.

THEOREM 5.1. For each graph $G$ with $n$ vertices and for $\lambda \in\langle-1,0\rangle$ it holds that

$$
m N_{\lambda}(G) \geqslant \frac{\sum_{i=1}^{n-1} i^{\lambda}}{\sum_{i=1}^{n-1} i^{1+\lambda}} .
$$

The bound is reached for a path (in its end-vertices).

Proof. If $\lambda \in\langle-1,0\rangle$ then minimal betweenness centrality (Theorem 4.2) and maximal transmission (Theorem 3.4) are both reached a path in its end-vertices and thus $m N_{\lambda}(G)$ is also reached for a path in its end-vertices.

A broom $B_{n, k}$ is a graph obtained by identification of a pendant vertex of star $S_{k+1}$ and an end-vertex of path $P_{n-k}$. The other end-vertex of the path is called starting vertex of the broom. In particular, $B_{n-1,2}=P_{n}$ and $B_{1, n}=S_{n}$. bellow.

We will need an auxiliary lemma which will be used in several proofs 
LEMMA 5.2. For positive numbers $a_{1}, a_{2}, \ldots, a_{n}, b_{1}, b_{2}, \ldots, b_{n}$ the following holds

$$
\frac{\sum_{i=1}^{n} a_{i}}{\sum_{i=1}^{n} b_{i}} \geqslant \min \left\{\frac{a_{i}}{b_{i}}\right\}
$$

Proof. It holds

$$
\frac{\sum_{i=1}^{n} a_{i}}{\sum_{i=1}^{n} b_{i}}=\frac{\sum_{i=1}^{n} b_{i} \cdot \min \left\{\frac{a_{i}}{b_{i}}\right\} \cdot \frac{\frac{a_{i}}{b_{i}}}{\min \left\{\frac{a_{i}}{b_{i}}\right\}}}{\sum_{i=1}^{n} b_{i}} \geqslant \frac{\sum_{i=1}^{n} b_{i} \cdot \min \left\{\frac{a_{i}}{b_{i}}\right\}}{\sum_{i=1}^{n} b_{i}}=\min \left\{\frac{a_{i}}{b_{i}}\right\} .
$$

THEOREM 5.3. For each graph $G$ with $n$ vertices and for $\lambda \in\langle-\infty,-1\rangle$ it holds that

$$
m N_{\lambda}(G) \geqslant \min _{2 \leqslant D \leqslant n-1} \frac{D^{\lambda}+\frac{1}{n-D} \sum_{i=1}^{D-1} i^{\lambda}}{D^{1+\lambda}+\frac{1}{n-D} \sum_{i=1}^{D-1} i^{1+\lambda}} .
$$

The bound is obtained for a broom (in its starting vertex).

Proof. Let $G$ be a graph for which the minimum of $m N_{\lambda}(G)$ is attained and let $u$ be a vertex of the graph for which $N_{\lambda}(u)=m N_{\lambda}(G)$.

We have

$$
N_{\lambda}(u)=\frac{c_{\lambda}(u)}{t_{\lambda}(u)} \geqslant \frac{\sum_{v \in V \backslash\{u\}} d(u, v)^{\lambda}}{\sum_{v \in V \backslash\{u\}} d(u, v)^{\lambda+1}}
$$

because $u$ certainly lies on every shortest path between itself and every other vertex $v$.

Now let $v_{D}$ be a vertex which is farthest away from $u$ and let $S=$ $u v_{1} v_{2} \ldots v_{D}$ be a shortest path from $u$ to $v_{D}$.

Furthermore, let $k=n-D-1$, let $\left\{w_{1}, \ldots w_{k}\right\}=V \backslash\left\{u, v_{1}, \ldots, v_{D}\right\}$ be set of all vertices that do not lie on the path $S$ and let $W=\left\{w_{1}, w_{2}, \ldots, w_{k}, v_{D}\right\}$.

Since $d\left(u, v_{i}\right)=i$ for all $i \in\{1,2, \ldots, D\}$, we have

$$
m N_{\lambda}(G)=N_{\lambda}(u) \geqslant \frac{\sum_{v \in V \backslash\{u\}} d(u, v)^{\lambda}}{\sum_{v \in V \backslash\{u\}} d(u, v)^{\lambda+1}}=\frac{\sum_{i=1}^{D} i^{\lambda}+\sum_{i=1}^{k} d\left(u, w_{i}\right)^{\lambda}}{\sum_{i=1}^{D} i^{\lambda+1}+\sum_{i=1}^{k} d\left(u, w_{i}\right)^{1+\lambda}}
$$


The last expression in (5.1) can be written in the following way

$$
\frac{\sum_{v \in W}\left(d(u, v)^{\lambda}+\frac{1}{n-D} \sum_{i=1}^{D-1} i^{\lambda}\right)}{\sum_{v \in W}\left(d(u, v)^{1+\lambda}+\frac{1}{n-D} \sum_{i=1}^{D-1} i^{1+\lambda}\right)} .
$$

Using Lemma 5.2 the minimum of expression given in (5.2) is

$$
\frac{x^{\lambda}+\frac{1}{n-D} \sum_{i=1}^{D-1} i^{\lambda}}{x^{1+\lambda}+\frac{1}{n-D} \sum_{i=1}^{D-1} i^{1+\lambda}}
$$

where $x=d(u, v)$ for some $v \in W$.

This minimum is obtained if and only if ratio $\frac{a_{i}}{b_{i}}$ is constant for all $i \in$ $\{1,2, \ldots, n\}$ and one way to achieve this in our case is that $d(u, v)$ is constant for all $v \in\left\{w_{1}, \ldots, w_{k}, v_{D}\right\}$, i.e. that $d\left(u, w_{i}\right)=d\left(u, v_{D}\right)=D$ for all $i \in$ $\{1,2, \ldots, k\}$. This is only possible if $w_{i}$ is directly connected to $v_{D-1}$ for all $i \leqslant k$, which proves that $G$ is indeed a broom.

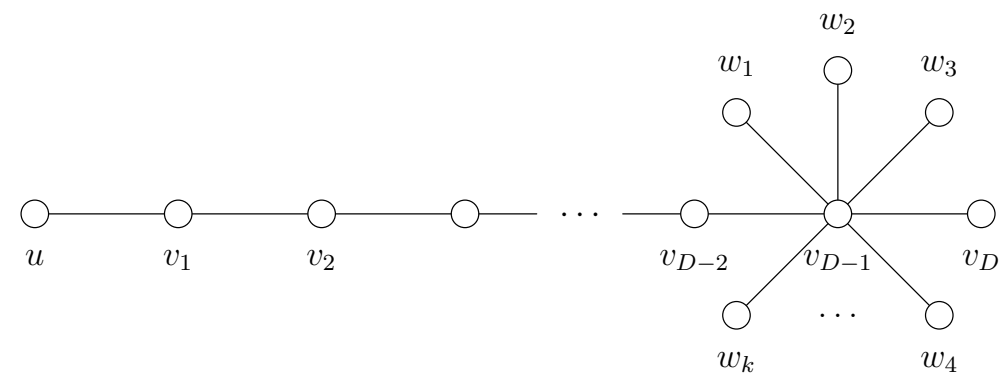

Figure 2. A broom that minimizes $m N_{\lambda}(G)$.

REMARK 5.4. Figure 2 shows that the graph that minimizes $m N_{\lambda}(G)$ is a proper broom for some combinations of $n$ and $\lambda$.

THEOREM 5.5. For each graph $G$ with $n$ vertices and for $\lambda<0$ it holds that

$$
m N_{\lambda}(G) \leqslant 1 .
$$

The bound is reached for any vertex of a vertex-transitive graph. 


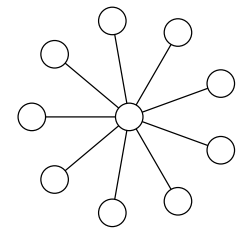

$\lambda=-4$

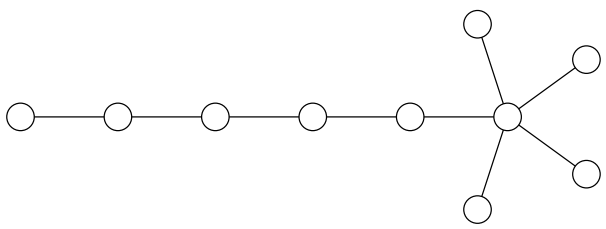

$\lambda=-1.6$

Figure 3. Graphs that minimize $m N_{\lambda}(G)$ for $n=10$ and two values of $\lambda$.

Proof. Using Lemma 2.1 and Lemma 5.2 we have

$$
\min \left\{\frac{c_{\lambda}(u)}{t_{\lambda}(u)}: u \in V\right\} \leqslant \frac{\sum_{u \in V} c_{\lambda}(u)}{\sum_{u \in V} t_{\lambda}(u)}=\frac{2 W_{1+\lambda}(G)}{2 W_{1+\lambda}(G)}=1 .
$$

\subsection{Maximal networkness.}

THEOREM 5.6. For each graph $G$ with $n$ vertices and for $\lambda<0$ it holds that

$$
1 \leqslant M N_{\lambda}(G) \leqslant 2^{\lambda}(n-2)+1 .
$$

The lower bound is reached for a vertex-transitive graph and the upper bound is reached for a star (in its central vertex).

Proof. Let us first prove the lower bound. Using Lemma 2.1 we have

$$
\max \left\{\frac{c_{\lambda}(u)}{t_{\lambda}(u)}: u \in V\right\} \geqslant \frac{\sum_{u \in V} c_{\lambda}(u)}{\sum_{u \in V} t_{\lambda}(u)}=\frac{2 W_{1+\lambda}(G)}{2 W_{1+\lambda}(G)}=1 .
$$

For the upper bound, let $u \in V$ be the vertex that maximizes networkness. From Lemma 4.1 it follows that the graph that maximises $M N_{\lambda}$ is a tree. Namely, since networkness is defined as quotient of betweenness centrality and transmission, we want the numerator to be maximal. That holds when the graph $G$ is a tree. We can assume that the denominator is also a tree. If this is not the case, we can repeat the construction of $G^{\prime}$ in Lemma 4.1 to obtain a tree in which the distances between $u$ and all the other vertices stay the same, thus, transmission stays the same. Using power mean inequality (for $x \leqslant y$ )

$$
\left(\frac{\sum_{i=1}^{n} a_{i}^{x}}{n}\right)^{\frac{1}{x}} \leqslant\left(\frac{\sum_{i=1}^{n} a_{i}^{y}}{n}\right)^{\frac{1}{y}}
$$


between powers 1 and $\lambda$ we have

$$
\begin{aligned}
N_{\lambda}(u)= & \frac{\sum_{q \in[u]} b_{\lambda}(u q)}{\sum_{v \in V \backslash\{u\}} d(u, v)^{1+\lambda}} \\
\leqslant & \frac{2 \sum_{\{v, w\} \in\left(\begin{array}{l}
V \backslash\{u\} \\
2
\end{array}\right)}[d(v, u)+d(u, w)]^{\lambda}+\sum_{v \in V \backslash\{u\}} d(u, v)^{\lambda}}{\sum_{v \in V \backslash\{u\}} d(u, v)^{1+\lambda}} \\
\leqslant & \frac{2 \sum_{\{v, w\} \in\left({ }^{V \backslash\{u\}}\right)}\left[d(v, u)^{\lambda}+d(u, w)^{\lambda}\right] \cdot \frac{2^{\lambda}}{2}+\sum_{v \in V \backslash\{u\}} d(u, v)^{\lambda}}{2_{v \in V \backslash\{u\}}^{\lambda}(n-2) \sum_{v \in V \backslash\{u\}} d(u, v)^{\lambda}+\sum_{v \in V \backslash\{u\}} d(u, v)^{\lambda}} \\
\leqslant & \frac{\left[2^{\lambda}(n-2)+1\right] \sum_{v \in V \backslash\{u\}} d(u, v)^{1+\lambda}}{\sum_{v \in V \backslash\{u\}} d(u, v)^{1+\lambda}} \leqslant 2^{\lambda}(n-2)+1 .
\end{aligned}
$$

A simple calculation shows that this bound is reached for a star in its central vertex.

\section{Network SURPLus}

\subsection{Minimal network surplus.}

THEOREM 6.1. For each graph $G$ with $n$ vertices and for $\lambda<0$ the following holds

$$
m \nu_{\lambda}(G) \leqslant 0 .
$$

The bound is reached for any vertex of a vertex-transitive graph.

Proof. Using Lemma 2.1 and the comparison of minimum and average of $c_{\lambda}(u)$, we obtain

$$
\begin{aligned}
m \nu_{\lambda}(u) & =\min \left\{c_{\lambda}(u)-t_{\lambda}(u): u \in V\right\} \leqslant \frac{1}{n}\left(\sum_{u \in V}\left(c_{\lambda}(u)-t_{\lambda}(u)\right)\right) \\
& =\frac{1}{n}\left(\sum_{u \in V} c_{\lambda}(u)-\sum_{u \in V} t_{\lambda}(u)\right)=\frac{1}{n}\left(2 W_{1+\lambda}(G)-2 W_{1+\lambda}(G)\right)=0 .
\end{aligned}
$$

The equality obviously holds for vertex-transitive graph. 
THEOREM 6.2. For each graph $G$ with $n$ vertices and for $\lambda \in\langle-\infty,-1\rangle$ the following holds

$$
m \nu_{\lambda}(G) \geqslant-(n-2) \cdot 2^{\lambda} .
$$

The bound is reached for a star (in its pendant vertex).

Proof. Let $u$ be a vertex such that $m \nu_{\lambda}(G)=\nu_{\lambda}(u)$. We have

$$
\begin{aligned}
\nu_{\lambda}(u) & =c_{\lambda}(u)-t_{\lambda}(u) \\
& \geqslant \sum_{v \in V \backslash\{u\}} d(u, v)^{\lambda}-\sum_{v \in V \backslash\{u\}} d(u, v)^{1+\lambda} \\
& =\sum_{v \in V \backslash\{u\}} d(u, v)^{\lambda}(1-d(u, v)) \geqslant-(n-2) \cdot 2^{\lambda},
\end{aligned}
$$

because function $f(x)=x^{l}(1-x)$ is increasing on $\langle 2,+\infty\rangle$ and $n-2$ is the maximum number of vertices $v \in V$ such that $d(u, v)=2$.

TheOREM 6.3. For each graph $G$ with $n$ vertices and for $\lambda \in\langle-1,0\rangle$ the following holds

$$
m \nu_{\lambda}(G) \geqslant \sum_{i=1}^{n-1}\left(i^{\lambda}-i^{1+\lambda}\right) .
$$

The bound is reached for a path (in its end-vertex).

Proof. If $\lambda \in\langle-1,0\rangle$ then minimal betweenness centrality (Theorem 4.2) and maximal transmission (Theorem 3.4) are both reached for an endvertex of a path and thus $m \nu_{\lambda}(G)$ is also reached for an end-vertex of a path.

\subsection{Maximal network surplus.}

THEOREM 6.4. For each graph $G$ with $n$ vertices and for $\lambda<0$ it holds that

$$
0 \leqslant M \nu_{\lambda}(G) \leqslant(n-1)(n-2) \cdot 2^{\lambda} .
$$

The lower bound is reached for a vertex-transitive graph and the upper bound is reached for a star (in its central vertex).

Proof. For lower bound, using Lemma 2.1 and the comparison of maximum and average of $c_{\lambda}(u)$, we obtain

$$
\begin{aligned}
M \nu_{\lambda}(u) & =\max \left\{c_{\lambda}(u)-t_{\lambda}(u): u \in V\right\} \geqslant \frac{1}{n}\left(\sum_{u \in V}\left(c_{\lambda}(u)-t_{\lambda}(u)\right)\right) \\
& =\frac{1}{n}\left(\sum_{u \in V} c_{\lambda}(u)-\sum_{u \in V} t_{\lambda}(u)\right)=\frac{1}{n}\left(2 W_{1+\lambda}(G)-2 W_{1+\lambda}(G)\right)=0 .
\end{aligned}
$$


Let us prove the upper bound. Analogously as in the proof of Theorem 5.6, from Lemma 4.1 it follows that the graph for which the upper bound is obtained is a tree. Let $u \in V$ be the vertex that maximizes $\nu_{\lambda}$. It holds

$$
\begin{aligned}
\nu_{\lambda}(u) & =c_{\lambda}(u)-t_{\lambda}(u) \\
& \leqslant \sum_{v \in V \backslash\{u\}} d(u, v)^{\lambda}+2 \sum_{v, w \in V \backslash\{u\}} d(v, w)^{\lambda}-\sum_{v \in V \backslash\{u\}} d(u, v)^{1+\lambda} \\
& \leqslant \sum_{v \in V \backslash\{u\}} d(u, v)^{\lambda}+2 \sum_{v, w \in V \backslash\{u\}} 2^{\lambda}-\sum_{v \in V \backslash\{u\}} d(u, v)^{1+\lambda} \\
& =\sum_{v \in V \backslash\{u\}} d(u, v)^{\lambda}+(n-1)(n-2) \cdot 2^{\lambda}-\sum_{v \in V \backslash\{u\}} d(u, v)^{1+\lambda} \\
& \leqslant(n-1)(n-2) \cdot 2^{\lambda} .
\end{aligned}
$$

\section{ACKNOWLEDGEMENTS.}

The partial support of the Croatian Ministry of Science, Education and Sports (Grant numbers 177-0000000-0884 and 037-0000000-2779) and EuroGIGA Collaborative Research Project GReGAS. We also thank anonymous referees for valuable advice and help.

\section{REFERENCES}

[1] J. M. Anthonisse, The rush in a directed graph, Stichting Mathematisch Centrum, Amsterdam, 1971, 1-10.

[2] A.-L. Barabási, Linked: How everything is connected to everything else and what it means, Persus Publishing, Cambridge, 2002.

[3] A. Barrat, M. Barthélemy and A. Vespignani, Dynamical processes on complex networks, Cambridge University Press, Cambridge, 2008.

[4] B. Bollobás, Modern graph theory, Springer-Verlag, New York, 1998.

[5] S. P. Borgatti and M. G. Everett, A graph-theoretic perspective on centrality, Social Networks 28 (2006), 466-484.

[6] U. Brandes, A faster algorithm for betweenness centrality, J. Math. Sociol. 25 (2001), $163-177$.

[7] U. Brandes and T. Erlebach (eds.), Network analysis - methodological foundations, Springer-Verlag, Berlin, 2005.

[8] G. Caporossi, M. Paiva, D. Vukičević and M. Segatto, Centrality and betweenness vertex and edge decomposition of the Wiener index, MATCH Commun. Math. Comput. Chem 68 (2012), 293-302.

[9] L. Freeman, A set of measures of centrality based on betweenness, Sociometry 40 (1977), 35-41.

[10] L. Freeman, Centrality in social networks: conceptual clarification, Social Networks 1 (1978), 215-239.

[11] S. Gago, J. Hurajová and T. Madaras, Nodes on the betweenness centrality of a graph, Math. Slovaca 62 (2012), 1-12.

[12] M. Girvan, M. E. J. Newman, Community structure in social and biological networks, Proc. Natl. Acad. Sci. USA 99 (2002), 7821-7826. 
[13] I. Gutman, A property of the Wiener number and its modifications, Indian J. Chem. 36 (1997), 128-132.

[14] I. Gutman, D. Vidović and Lj. Popović, On graph representation of organic molecules - Cayley's plerograms vs. his kenograms, J. Chem. Soc. Faraday Trans. 94 (1998), 857-860.

[15] M. E. J. Newman, Networks. An introduction, Oxford University Press, Oxford, 2010.

[16] D. Vukičević and G. Caporossi, Network descriptors based on betweenness centrality and transmission and their extremal values, submitted to Discrete Appl. Math.

[17] H. Wiener, Structural determination of paraffin boiling points, J. Amer. Chem. Soc. 69 (1947), 17-20.

S. Antunović

Faculty of Civil Engineering, Architecture and Geodesy

University of Split

Matice hrvatske 15, 21000 Split

Croatia

E-mail: santunovic@gradst.hr

T. Kokan

Faculty of Economics

University of Split

Cvite Fiskovića 5, 21000 Split

Croatia

E-mail: tkokan@gmail.com

T. Vojković

Department of Mathematics

University of Split

Teslina 12, 21000 Split

Croatia

E-mail: tanja@pmfst.hr

D. Vukičević

Department of Mathematics

University of Split

Teslina 12, 21000 Split

Croatia

E-mail: vukicevic@pmfst.hr

Received: 18.7.2012.

Revised: 25.2.2013. 